\title{
Proposal of BCM Evaluation Method Based on Disaster Scenario Simulation
}

\author{
Ryuhei Kaneko and Yoshio Nakatani \\ Graduate School of Engineering Science, Ritsumeikan University, \\ 1-1, Noji-Higashi 1, Kusatsu, Shiga 525-0058, Japan \\ cc005063@ed.ritsumei.ac.jp, nakatani@is.ritsumei.ac.jp
}

\begin{abstract}
Almost $20 \%$ of big earthquakes in the world occur in Japan. It becomes impossible for private companies and governments to carry out their business if they suffer from severe damage. In this situation, BCM (Business Continuity Management) become focus of public attention. But it has not become popular enough. This is due to the fact that anti-disaster plan is difficult to be verified because companies are lack of knowledge about what situations may occur in disasters. This research proposes how situation changes in companies by using a disaster progress simulator and actual cases when disasters happen. By using this system, companies can study what situation changes and clarify problems of their BCMs. As a result, the companies can settle on more realistic BCMs and carry out business even in disasters.
\end{abstract}

Keywords: Business Continuity Management, disaster simulation.

\section{Introduction}

A lot of earthquakes occur in Japan. It becomes impossible for private companies, social infrastructure providers, such as electric power companies, gas companies, network companies, and road/railway companies, and governments to carry on their operations when they suffer from severe damages in big earthquakes. In this situation, BCM (Business Continuity Management) become the focus of public attention in Japan. BCM is the activity that settles on policies and procedures to achieve business continuance of companies in contingencies such as the natural damage and terrorism. Currently, Japanese companies are growing interest in BCM, especially after the 9.11 terrorism. But it is not popular enough. This is due to the fact that anti-disaster plan is difficult to be verified because companies are lack of knowledge about what situations may occur in disasters.

This research proposes an anti-disaster planning support system based on disaster scenario simulation which simulates what kind of situations may occur about companies when a disaster occurs. Then this research aims to provide the companies with an efficient environment to investigate BCM enough. 


\section{BCM}

\subsection{What Is BCM}

This system supports BCM of companies and governments. BCM is realized by BCP (Business Continuity Plan), which is an action plan of BCM. BCP is required to improve on regular according to changing conditions and knowledge accumulation about disasters because BCP should be effective. The PDCA (Plan Do Check Action: PDCA) cycle has to be rigorously executed as a method of improving them continuously.

Figure 1 reveals that companies innovating through $\mathrm{BCM}$ can improve the expedition of their re-establishment and rundown of their capacity operating rate [1].

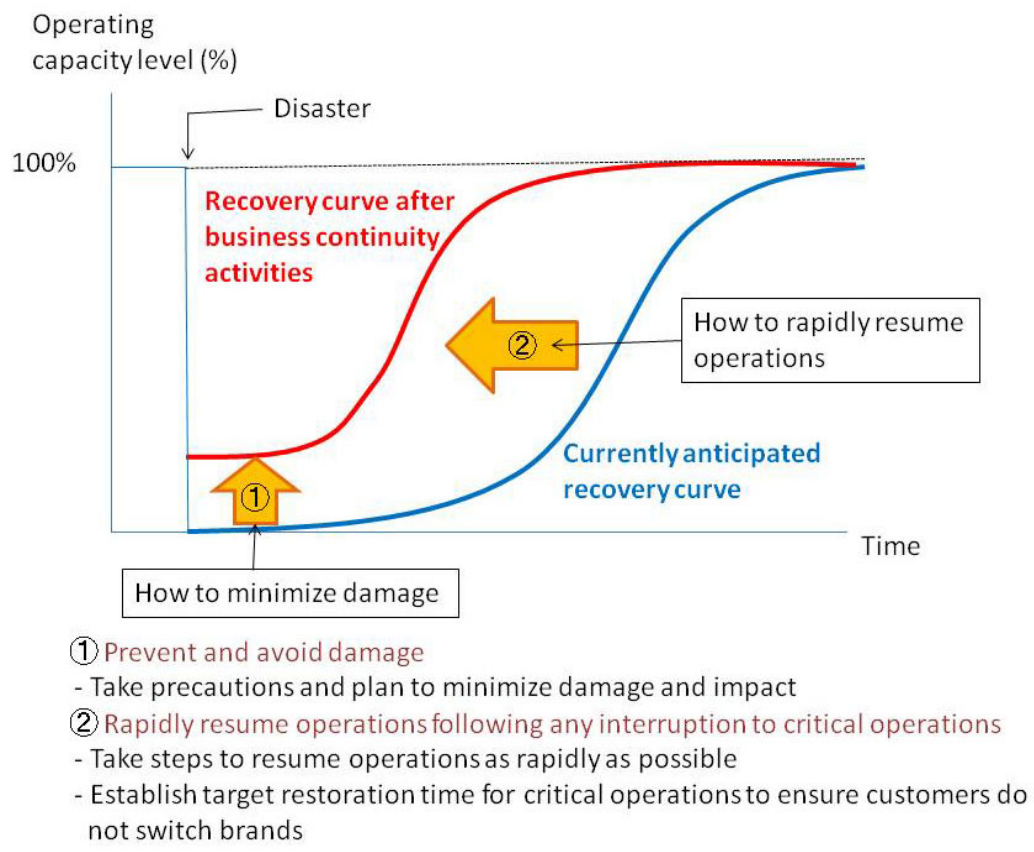

Fig. 1. Effect of BCM [1]

\subsection{Actual Conditions Regarding BCM}

In Japan the numbers of company realizing necessity of BCMs are increasing. The numbers of companies settling on BCMs are also increasing. Therefore, it can be increase the company having interest in $\mathrm{BCP}$.

However, BCP can be made more effectively by regularly reviewing and improving it. The figure. 2 gives the number of Japanese companies that don't have much training about BCPs [2]. 


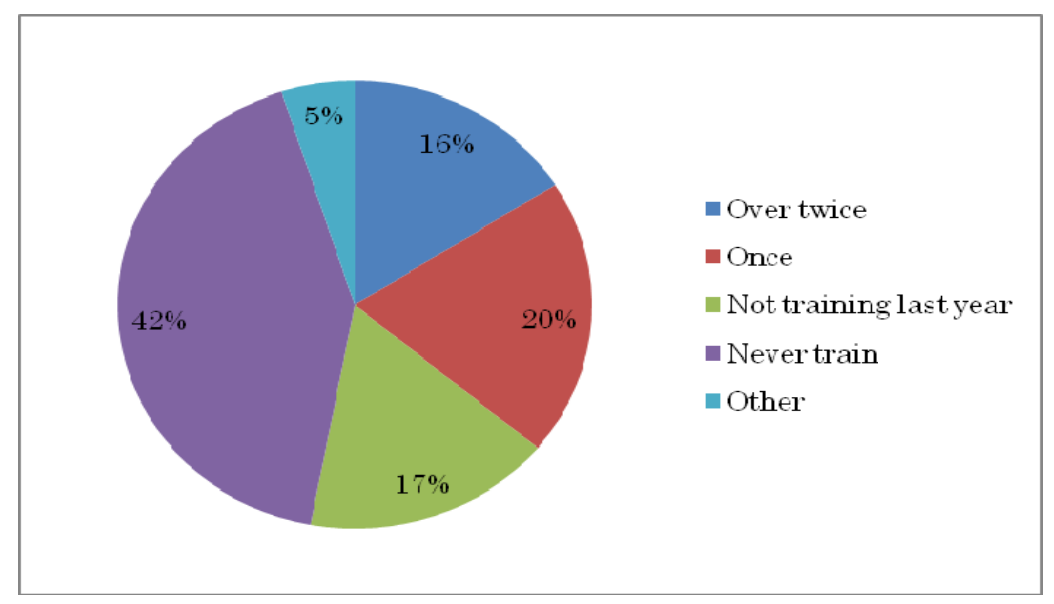

Fig. 2. Graph of BCM education and study

\subsection{Related Researches}

Research and development of BCM support is currently an attractive and important field and hence the amount of research on it is gradually increasing, although still can be said to be insufficient. An example of the research on supporting BCMs is an earthquake emergency response scenario simulator [3]. It suggests damage situation based on an assuming earthquake scenario. It utilizes a visual imaging database to provide support for making judgments and decisions during the state of emergency after an earthquake. Then a user can plan disaster recovery from damage situation.

\subsection{Problems on Supporting BCM}

BCMs in Japan include several problems, details on which are provided below.

The number of companies that realizes necessity of BCM and settles on BCM is increasing. However, many companies don't continue to improve and revise BCMs after formulating them. One reason is a problem with difficulty in verification whether they are useful and comprehensive even if BCM is formulated. Especially companies have apprehensions about what situation may break out, even if companies can consider various kinds of countermeasures, based on lack of knowledge.

This apprehension is not reduced enough by previous researches. The computer simulation is effective to call up an image of situation changes in a specific scene, but it is difficult for a company to understand the entire damage that may be caused by a certain level of disaster.

\section{Prototype System}

\subsection{System Summary}

This research proposes the system that provides a company with a whole image of possible damage. 
This system simulates the development of damages, pointing out what changes may take place in which human resource and equipment of a company, based on general knowledge on causal chain of damages. The user select a part of causal chain by selecting human resource and equipment of his/her company as a initial data, from a list of possible resources that may exist in an average company of a certain area. This causal chain doesn't consider a temporal element, and state transition from damage on a resource to damages on other resources is reasoned. However, the user can assume how long a state does on, because a temporal element is essential in disaster.

System architecture is shown in Figure.3.

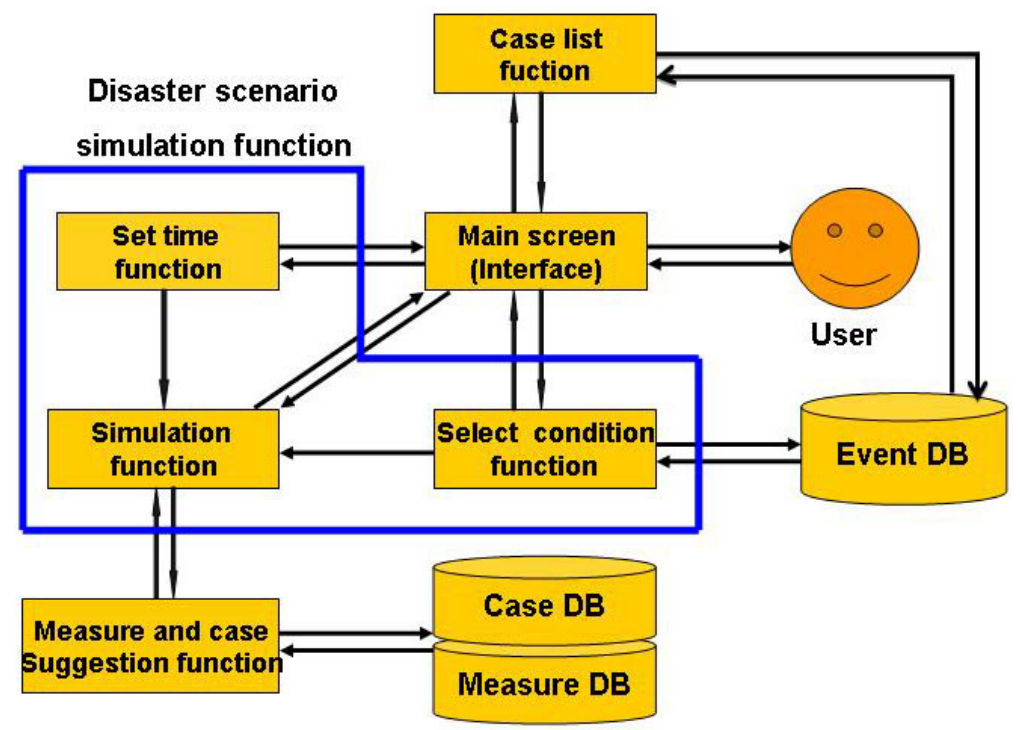

Fig. 3. Image of system architecture

A company can rate the BCM. This system provides the user with functions that change the setting of simulation: "condition selection function" that selects resources from a list of possible resources, "time-setting function" that specifies how long a state goes on, "simulation function" that executes simulation based on specified conditions, and "measure and case suggestion function" that suggests measures and suggests related previous cases.

This system was implemented with Java and PostgreSQL on Windows OS.

\subsection{Data Form}

This system holds several databases to store the data of events, cases and measures.

1. Event selection database. This database is for which conditions are selected. The data include the basic attributes of a company: building (office/factory) and location (city part/mountainous district/sea surrounding/innings). The assumed hours of simulation is also specified (morning commuting hours/ business hours/ evening commuting hours). 
2. Detailed event database. Event names, their IDs and categories are stored. Several events that may occur on certain resources in certain disasters are stored. Resources are described in a level of general categories, such as PC (personal computers) and telecommunications equipment.

3. Case database. Details of the previous cases (places, categories, preceding events, and dates when the cases occurred) are stored in this database. The preceding events are used as conditions that triggered the case.

4. Measure database. Measures to possible events are stored.

\subsection{Condition Selection Function}

This function can allow setting of the basic condition of a company and assumed hours of simulation. The system selects the appropriate events from the detailed event database based on the selected conditions. Then the system selects the events succeeding the selected events in the database. This selection continues until the appropriate events are not found.

When the system selects the events, the same events are selected every time, because the conditions are the same. The system selected the events randomly among the candidate events.

Figure. 4 is the examples of select condition function.

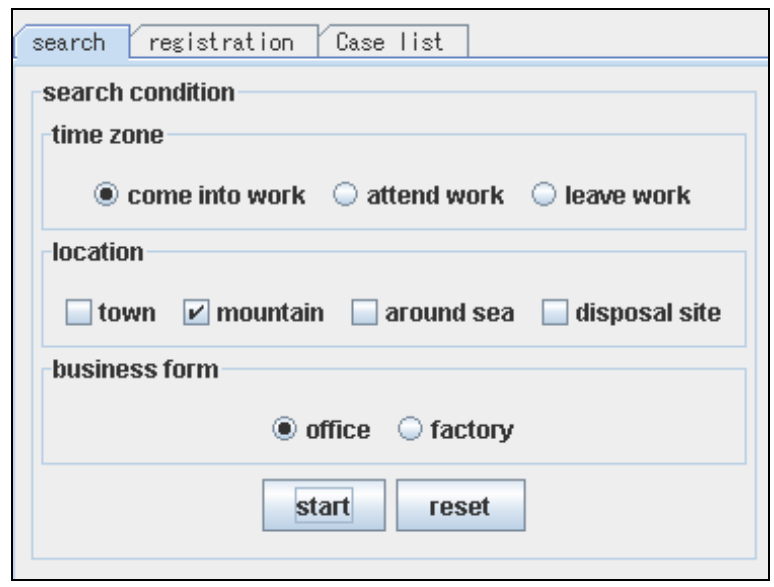

Fig. 4. An example of the condition selection function

\subsection{Time-Setting Function}

The system holds the data as a common scenario for the development of disaster, but it doesn't consider temporal element of how long each event goes on. Therefore the user can set the duration to each event.

First, the user selects the event that he/she wants to set duration. Next, the system proposes possible durations that the user can select based on the relationship between the preceding and succeeding events. The possible duration is proposed based on the end time of the preceding event and the start time of the succeeding events. The users 
can set the duration within the proposed period. Then the system shows the duration in the Gantt-chart on the screen.

Figure. 5 is an example of the time-setting function.

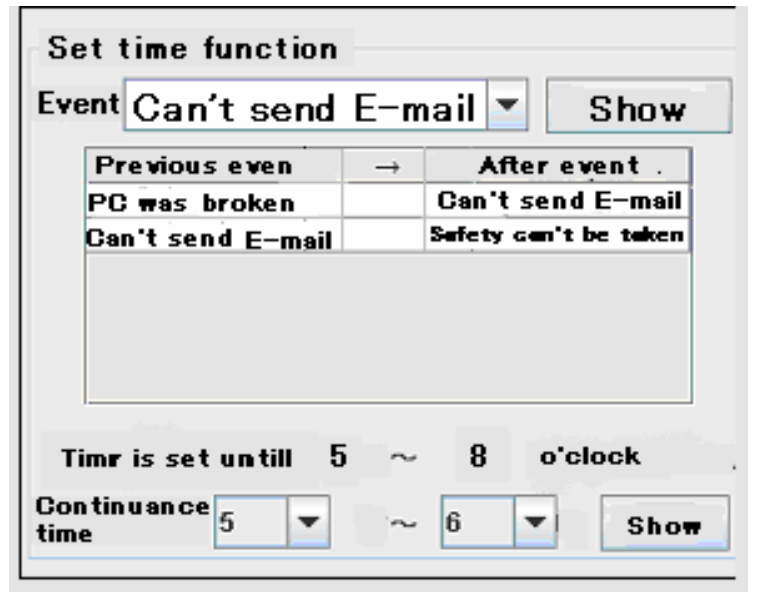

Fig. 5. An example of the time-setting function

\subsection{Simulation Function}

This function is the main function. This function simulates the development of damages based on the specified conditions and selected events. When the user pushes "simulation button," simulation starts.

The system follows the event chain in the detailed event database and randomly selects some of the appropriate events, until the succeeding events are not found. For

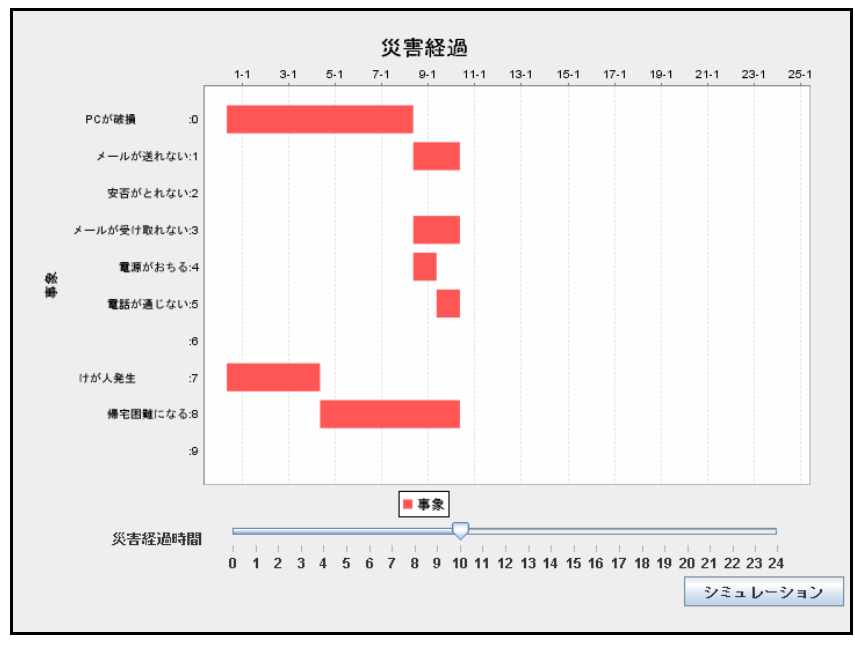

Fig. 6. An example of the simulation function 
example, the system reasons "damage of PC falling in the water" based on the transit from "quake" to "falling of PC" and another transit from "quake" to "damage of a water pipe." The system further reasons "impossible to send e-mails" based on "damage of PC falling in the water." The result is shown in the Gantt-chart.

Figure.6 is an example of the simulation function.

\subsection{Measure and Case Suggestion Function}

This function is another main function. The disaster scenario simulation gives the user the damage image but the user doesn't know how to take countermeasures against possible damages. This function suggests a related previous case and a possible countermeasure. The case shows "what happened at other company when the event occurred" and "what caused the event." In addition to this, the system shows a possible common countermeasure in the measure database.

This function supports revision and improvement of the BCP.

Figure.7 is an example of the measure and case suggestion function.

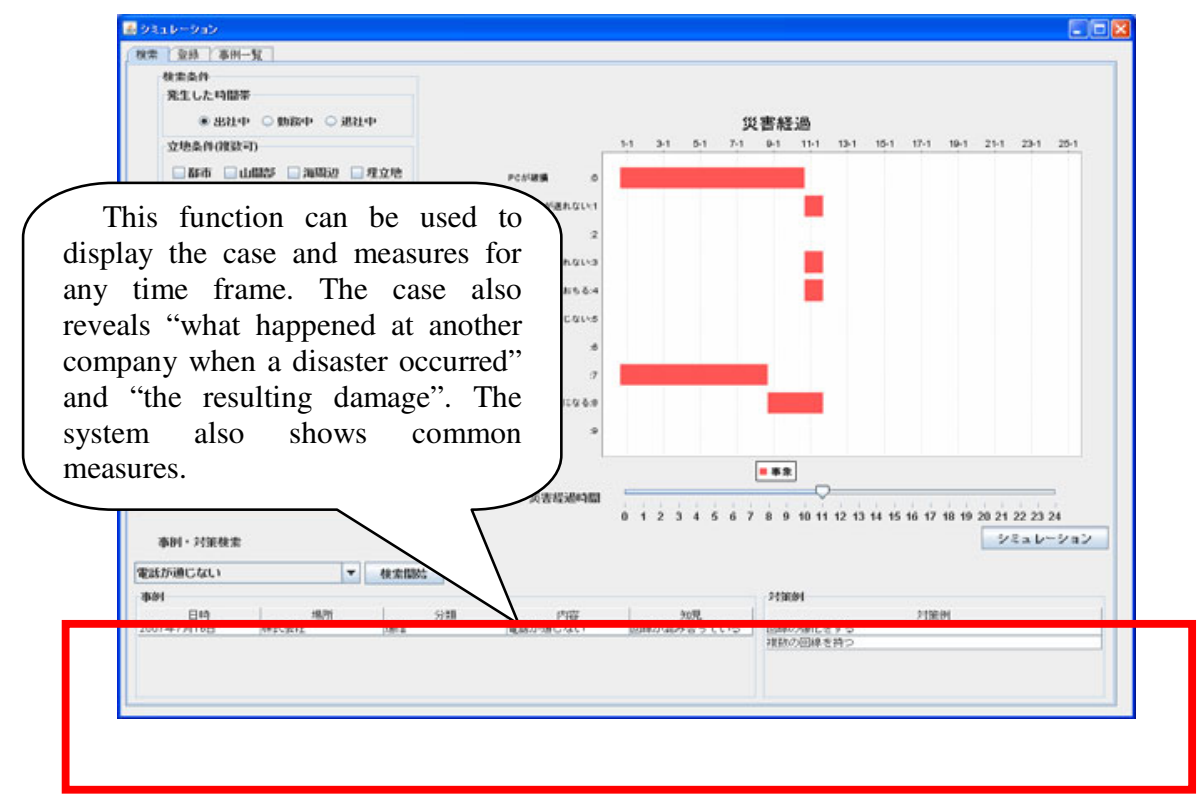

Fig.7. An example of the measure and case suggestion function

\section{Result}

The systems were evaluated by 2 presidents of a software development company and an insurance company in Japan.

The scenario situation simulator and the measure and case suggestion function were evaluated. 


\subsection{Evaluation Regarding Scenario Simulation Function}

- It is a simple structure, easy to determine what has occurred

- The user can image whole disaster but is difficult to understand the relationship between events.

- Events should be shown by classified into their categories for easiness to understanding.

- It is necessary to show the event dividing into two frames of local risks and common risks.

The scenario simulation function has strictly opinions. In the future, how to show the event is considered.

\subsection{Evaluation Regarding Measure and Case Suggestion Function}

1. This function realizes continuous revision of the $\mathrm{BCP}$ by considering the previous cases and common countermeasures.

2. In accordance with the division of events by categories, the cases and countermeasures should also be classified into categories.

This function is effective but it is necessary to consider how to show the events and cases in accordance with the scenario simulation function. It is necessary to support $\mathrm{BCP}$ more effectively. Therefore the system will have a function about rate and support BCP.

\section{Conclusion}

This research proposes the damage scenario simulation to support revision of BCP in term of what damage may occur and how the damage develops.

The evaluation of the prototype system by the two entrepreneurs shows some good result but some improvement is pointed out. In the next step, improvement will be considered. The system should be evaluated by installed at company. Through actual use, the system has to be improved in the future.

\section{References}

1. NEC: Approach to Business Continuity, http://www. nec.co.jp/csr/en/report2008/continued/index.html (referred February 3, 2011)

2. NTT-AT: Consider consulting to help implement recovery measures precaution measures "Business Continuity Planning Consulting Services, https://www.nttat.co.jp/product/bcp/index.html (referred August 29, 2009)

3. Naganoh, M., Taniai, M., Ikeda, J.: Earthquake Emergency Response Scenario Simulator. Computational Studies A 2, 157-167 (1998) 